\title{
Frequency drift during intensive SSFP scanning: implications and solution for 3T neonatal CMR
}

\author{
Anthony N Price ${ }^{1,2^{*}}$, Shaihan J Malik ${ }^{1,2}$, Kathryn Broadhouse ${ }^{2}$, Anna Finnemore ${ }^{2}$, Giuliana Durighel ${ }^{2}$, David J Cox, \\ AD Edwards ${ }^{1,2}$, Alan Groves $^{1,2}$, Joseph V Hajnal ${ }^{1,2}$ \\ From 16th Annual SCMR Scientific Sessions \\ San Francisco, CA, USA. 31 January - 3 February 2013
}

\section{Background}

Balanced-SSFP is widely used because of its inherent highcontrast and high-SNR efficiency, and therefore is an obvious choice for neonatal cardiac applications. Typically, multiple averages are needed due to the high-spatial and temporal resolutions required, especially for the smallest preterm infants. Therefore, prolonged intensive scans are required placing high demands on scanner hardware. Consequently, one of the two critical prerequisites for successful SSFP - sufficient B0 shimming and stable scanner frequency - is often not met using standard protocols.

\section{Methods}

Frequency drifts were assessed, alongside a method of active correction, at $3 \mathrm{~T}$ on phantoms and in vivo. A prolonged SSFP protocol, optimised for neonates, consisted of a multi-2D cine stack, retrospectively-gated to 20 cardiac phases, $1 \times 1 \mathrm{~mm}$ resolution in-plane $(\mathrm{FOV}=10 \times 10 \mathrm{~cm})$, $4 \mathrm{~mm}$ slices (10 with $\sim 1 \mathrm{~mm}$ overlap) and 8 averages (TA 10 mins, FA/TE/TR=35/1.9/3.8 ms). In phantoms an additional shim offset was applied to introduce band artifacts and allow visualisation of frequency drift.

Additional tests were performed using 3D SSFP acquired at $1 \times 1 \times 1 \mathrm{~mm}(\mathrm{FOV}=10 \times 10 \times 3 \mathrm{~cm}), 20$ phases, 1 average, TA 5 mins and repeated several times to assess whether drifts also occur in 3D protocols.

An active frequency stabilisation (FS) method was adapted and implemented into the multi-2D sequence. FS was achieved by monitoring phase differences between two gradient echo readouts applied between every slice increment, and compared to baseline. The FS stack protocol was performed on 18 infants (CGA: $27+5$ to $40+3$ weeks, weight: 610 to $3120 \mathrm{~g}$ ). B0 mapping and

1Division of Imaging Sciences and Biomedical Engineering, King's College London, London, UK

Full list of author information is available at the end of the article

image based shimming was used to ensure adequate B0 uniformity over the neonatal heart.

\section{Results}

The phase-slice reformats in Figure 1 reveal progressive drift in bands, and thus frequency, up through the
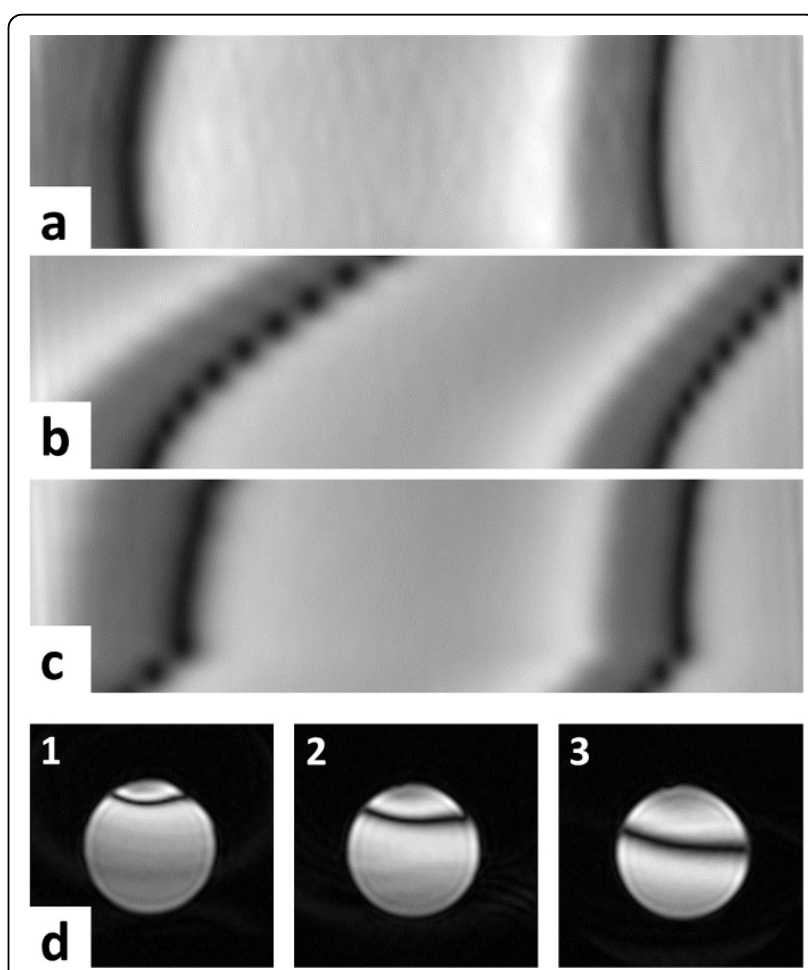

Figure 1 Frequency drift test in phantoms during prolonged SSFP: reformatted views (phase-slice direction) of a multi-2D stack acquisition in (a) fast reference scan - no drift, (b) neonatal optimised protocol and (c) added frequency stabilisation. Single slice view from (d) a 3D cine SSFP protocol also show a progressive frequency drift through repeated scan acquisitions 1-3. 

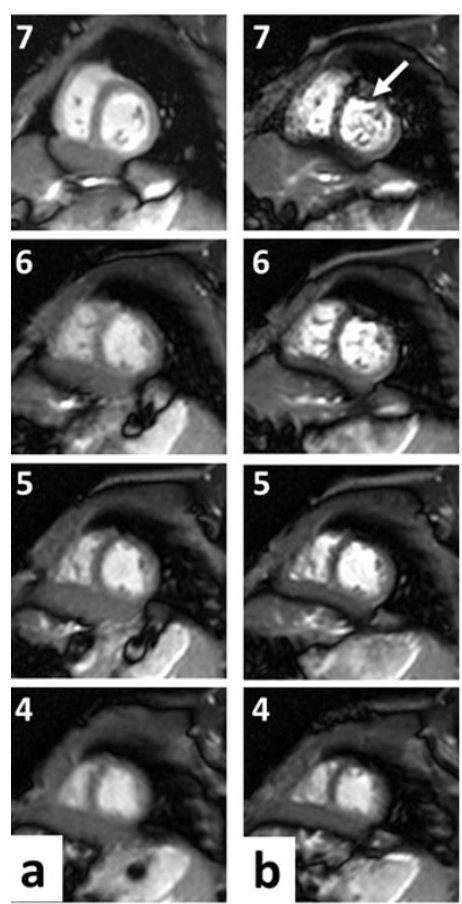

Figure 2 Neonatal cine SSFP stack acquisition (showing slices 4-7 from 10, apex-base order) (a) with and (b) without the proposed frequency stabilisation. The accumulated frequency drift updates performed during stack acquisitions in 13 infants is shown (c), the continuous black line represents the mean and the dashed line the 95\% confidence intervals for each FS update/slice increment.

prolonged SSFP stack protocol, compared to the reference scan. Repeating this with frequency stabilisation shows bands following a similar pattern to the reference from slice 3 onwards - suggesting stabilised frequency. Drifts were also observed using the 3D protocol between each repetition of the scan (TA 5mins). The in vivo example in Figure 2 reveals frequency drift severely degrading images by slice 7 , but rectified using frequency stabilisation. Artifact-free cine stacks were acquired in 16 infants, the frequency drift observed over 13 cases (Figure 2c) reveals an average drift of $165 \mathrm{~Hz}$, over the 10-minute protocol.

\section{Conclusions}

Significant drifts were found on a 3T system hampering the use of prolonged SSFP for neonatal CMR. Active frequency stabilising was achieved, vastly improving image quality and allowing for adequate volume segmentation. Frequency drifts may also affect adult CMR protocols requiring prolonged SSFP, such as free-breathing wholeheart cine or MRA.

\section{Funding}

MRC Clinician Scientist Fellowship

\section{Author details}

${ }^{1}$ Division of Imaging Sciences and Biomedical Engineering, King's College London, London, UK. ${ }^{2}$ Robert Steiner MRI Unit, Imaging Sciences

Department, Imperial College London, London, UK.

Published: 30 January 2013

doi:10.1186/1532-429X-15-S1-P5

Cite this article as: Price et al:: Frequency drift during intensive SSFP scanning: implications and solution for $3 \mathrm{~T}$ neonatal CMR. Journal of Cardiovascular Magnetic Resonance 2013 15(Suppl 1):P5.
Submit your next manuscript to BioMed Central and take full advantage of:

- Convenient online submission

- Thorough peer review

- No space constraints or color figure charges

- Immediate publication on acceptance

- Inclusion in PubMed, CAS, Scopus and Google Scholar

- Research which is freely available for redistribution
Biomed Central 